\title{
Deliberative Innovationen in Japan angesichts demokratischer Herausforderungen
}

\author{
Momoyo Hüstebeck
}

Online publiziert: 3. Dezember 2019

(C) Der/die Autor(en) 2019

Zusammenfassung Wie anderen etablierten Demokratien wird auch Japan eine Demokratiekrise attestiert. Das politische System weist neben der unter der jetzigen Regierung im Fokus stehenden Beschneidung von Freiheitsrechten weitere Demokratieschwächen wie eine geringe Wahlbeteiligung oder eine mangelnde horizontale Gewaltenkontrolle auf. Um der (partiellen) Krise der repräsentativen Demokratie zu begegnen, wird in der deutsch- sowie englischsprachigen Forschung die Implementation von demokratischen Innovationen diskutiert. Sie werden hier als topdown implementierte innovative Beteiligungsformen an institutionellen politischen Entscheidungsfindungen verstanden. Entsprechend der normativen Erwartung, der Demokratiekrise mit demokratischen Innovationen entgegenwirken zu können, wird deren Beitrag zur Demokratiequalität im vorliegenden Artikel am Beispiel Japans untersucht. Qualitative Evaluationskriterien aus Konzepten zur Demokratiequalität und demokratischen Innovationen finden dabei Anwendung auf japanische Fallbeispiele. Aus der Vielzahl von innovativen Partizipationsverfahren, die auch in Japan insbesondere Kommunen seit den 1990er Jahren implementierten, werden exemplarisch zwei deliberative Methoden evaluiert. Lokale Planungszellen (mini-publics) als eines der hier betrachteten Verfahren sind die weitverbreitetste Deliberationsform in Japan. Die erste nationale Deliberative Poll von 2012 dient als zweites Fallbeispiel. Die japanische Regierung initiierte sie unter dem Eindruck des Reaktorunfalls, um ein öffentliches Meinungsbild zur zukünftigen Energiestrategie Japans zu erhalten. Anhand der empirisch herausgearbeiteten demokratischen Stärken und Schwächen lässt sich folgern, dass demokratische Innovationen zwar als zusätzliche Partizipa-

BMBF-Projekt: Deliberation als innovative Form von demokratischer Bürgerbeteiligung im japanisch-deutschen Vergleich (FKZ:01UL1828X)

Dr. rer. pol. M. Hüstebeck $(\bowtie)$

IN-EAST Institut für Ostasienwissenschaften/Institut für Politikwissenschaft, Universität

Duisburg-Essen, Duisburg, Deutschland

E-Mail: momoyo.huestebeck@uni-due.de 
tionsverfahren punktuell die Qualität einer repräsentativen Demokratie (wie z.B. durch das Empowerment von Bürgern) verbessern, jedoch in ihrer Wirkung als ein Allheilmittel gegen die Demokratiekrise beschränkt sind.

\title{
Deliberative innovations in Japan against the backdrop of democratic challenges
}

\begin{abstract}
Like other established democracies, Japan has faced democratic challenges. Besides increased constraints on liberal democratic rights under the current national government, other democratic weaknesses, such as a record-low voter turnout or lacking checks and balances, have persisted in the Japanese political system. To counteract this (partial) crisis, democratic innovations have been in academic favor. A democratic innovation is here defined as a top-down implemented innovative institution of citizen involvement in policy-making. Based on the normative expectations of positive effects on democracy, this article investigates the impact of democratic innovations on the quality of democracy in Japan. For my evaluation, I apply qualitative criteria which refer to concepts of the quality of democracy, deliberation and democratic innovations. Choosing from a wide range of democratic innovations implemented, in particular at the local level since the 1990s, this study specifically scrutinizes two deliberative forms of participation. Namely, planningcells (mini-publics), as the most widely spread deliberative method in Japan, and the First National Deliberative Poll in 2012 serve as empirical cases. In the latter deliberative experiment, a randomly selected representative sample of citizens from all over Japan discussed and formed their opinions about Japan's future energy strategies after the nuclear accident. By qualitatively evaluating the two methods, this paper highlights their merits und challenges for strengthening democracy. Concluding from the empirical results, I ascertain some punctual improvements (such as citizen empowerment). However, implementing democratic innovations is not the universal remedy for curing the democratic malaise.
\end{abstract}

\section{Partizipative Entscheidungsverfahren für mehr demokratische Qualität}

Die japanische repräsentative Demokratie kennzeichnen eine niedrige Wahlbeteiligung, ein Vertrauensverlust in die politische Elite und eine Beschneidung von liberalen Bürgerrechten. So wird dem japanischen politischen System - wie anderen etablierten Demokratien - in wichtigen Bereichen der politischen Repräsentation und Legitimität eine Krise attestiert. Um den empirischen Schwächen der repräsentativen Demokratie zu begegnen, entwerfen Studien diesbezüglich unermüdlich neue, normative Konzepte für mehr Bürgermitbestimmung (Saward 2000; Geissel und Newton 2012; Steiner 2012, S. 27; Offe 2003, S. 18). Nicht nur in der Demokratietheorie, sondern auch in der politischen Praxis ist seit den 1990er Jahren ein weltweiter Trend zu innovativen Beteiligungsverfahren zu erkennen, die konventionelle Mitbestimmungsverfahren wie vornehmlich Wahlen ergänzen sollen. 
Die neuen Partizipationsformen werden im englisch- und deutschsprachigen Kontext unter dem Schlagwort ,demokratische Innovationen“ subsumiert. Das Konzept demokratischer Innovationen leitet sich von Theorien zur partizipativen und deliberativen Demokratie ab. An ihre Implementierung ist die Erwartung geknüpft, dass sie die repräsentative Demokratie von ihrer Malaise kurieren. Demokratische Innovationen sollen der Demokratiekrise entgegenwirken, indem sie die politische Kontrolle durch die Repräsentierten sowie die Legitimität der Repräsentanten verbessern (Hendriks 2005, S. 88, 91; Newton 2012, S. 7; Geissel 2012, S. 165; Tsubogō 2015, S. 168; Smith 2009, S. 2-4).

Aus der Vielzahl von innovativen Beteiligungsverfahren wurden für die vorliegende Analyse Deliberationen gewählt, da die japanische Forschungsliteratur diese als Instrument gegen die Demokratiekrise am meisten diskutiert. Andere Partizipationsmethoden wie eine Erweiterung der direkten Demokratie, die in anderen Ländern gerne propagiert wird, finden kaum Beachtung. Japanische Wissenschaftler plädieren dafür, den subnationalen Referenden durch ein verbindliches Bürgervotum mehr Gewicht zu verleihen, jedoch nicht für eine Implementierung dieser auf der nationalen Ebene. Die Forderung nach nationalen Plebisziten wird zu sehr mit dem Vorstoß von Ministerpräsident Abe Shinzō konnotiert, die pazifistische Verfassung zu ändern. Der in Artikel 9 der Nachkriegsverfassung verbriefte Kriegsverzicht ist so zentral für deren Perzeption und so tiefgreifend im Demokratieverständnis verankert, als dass direktdemokratische Innnovationen auf nationaler Ebene zur Disposition ständen. Dies erklärt, warum sich die japanische Partizipationsforschung vorrangig deliberativen Innovationen widmet.

Deliberationen werden hier als öffentliche, institutionalisierte Gruppendiskussionen zwischen Bürgern verstanden. Staatliche Institutionen organisieren diese und geben das Diskussionsthema vor. Verfahrensregeln gewähren indes idealiter den Bürgern einen von staatlicher Kontrolle freien Raum, in dem sie sich gleichberechtigt austauschen und eine eigene Meinung bilden können. Deliberationen stoßen ebenso soziale Lern- und Interaktionsprozesse an. Der rationale Austausch schafft konsensund damit gemeinwohlorientierte Ansätze, die der institutionalisierten Politik neue, zum Teil innovative Inputs bieten (Shinohara 2012; Landwehr 2012; Tamura 2017).

Diese Studie zu demokratischen Innovationen nimmt auf die normativen Deliberationskonzepte Bezug, wählt aber zugleich durch ihre Einbettung in den Diskurs um die Demokratiequalität einen empirischen Zugang. Dafür wird am Beispiel der japanischen Demokratie folgenden Fragen nachgegangen: Wo liegen im Hinblick auf eine demokratische Qualitätsverbesserung die Stärken und Schwächen von deliberativen Verfahren? Sind Deliberationen ein effektives Instrument, um den demokratischen Herausforderungen der repräsentativen Demokratie zu begegnen?

Für eine qualitative Bewertung werden Evaluationskriterien aus anderen Studien zur Demokratiequalität sowie zu demokratischen Innovationen zunächst extrahiert und dann zusammengeführt. Die konzeptionelle Grundlage dient dazu, die empirischen Grenzen und Chancen von demokratischen Innovationen für die japanische Demokratie zu beleuchten. Exemplarisch werden zwei Deliberationsmethoden lokale Planungszellen und die erste nationale Deliberative Poll - untersucht. Planungszellen sind in Japan die am weitesten verbreitete Deliberationsform. Deren Implementationen durch die Stadt Mitaka als erste japanische Kommune dienen 
hier als Fallbeispiele. Als Zweites wird die erste nationale Deliberative Poll in Japan analysiert. Während dieser diskutierten und stimmten Bürger im Sommer 2012 (16 Monate nach dem Reaktorunfall) über verschiedene Energieszenarien ab. Für die Fallstudien wurden deren ausführliche Dokumentationen durch die Organisatoren sowie die offiziellen Analysen ausgewertet. In einem zweiten Schritt wurden die Fallstudien mit denen weiterer Deliberationsbeispiele sowohl in Japan als auch andern Ländern verglichen.

\section{Herausforderungen der repräsentativen Demokratie in Japan}

Um die Chancen und Grenzen, die mit einer Implementierung von demokratischen Innovationen einhergehen, beurteilen zu können, werden in diesem Kapitel die Schwächen der japanischen repräsentativen Demokratie schlaglichtartig beleuchtet:

Erstens drückt sich ein tiefgreifender Vertrauensverlust in die politische Elite in einer sehr niedrigen Wahlbeteiligung aus. Annähernd nur fast jeder zweite Wahlberechtigte nimmt an der verbreitetsten politischen Partizipationsform teil. Die bis dato niedrigste Beteiligung von 59,3\% bei den Unterhauswahlen 2012 verdeutlichte die Unzufriedenheit der Wähler mit dem politischen Krisenmanagement der Dreifachkatstrophe (Watanabe und Schmidt 2013, S. 12-14). Im Folgenden fiel die Wahlbeteiligung weiter auf 52,66\% (2014) und 53,68\% (2017) (Akarui senkyo suishin kyōkai 2016a). Als ein geeignetes Instrument, dem Vertrauensverlust in die politischen Institutionen entgegenzuwirken, werden demokratische Innovationen erachtet. Die Partizipation lässt die Teilnehmenden positiv erfahren, dass sie ,politisch etwas bewegen“ können. Demokratische Innovationen wirken damit der verbreiteten Politikverdrossenheit auf der individuellen Ebene entgegen und initiieren oftmals eine weitere politische Teilhabe.

Zweitens wird die Gesamtbevölkerung durch die tatsächliche Wählerschaft in ihrer sozialen Zusammensetzung statistisch nicht gänzlich abgebildet. Bei japanischen Wahlen ist die niedrige Beteiligung der jungen Generation frappierend. Lediglich 32,58 \% der Zwanzig- bis Dreißigjährigen gingen bei den Unterhauswahlen 2014 und 33,85\% in 2017 zur Wahl. Damit liegt ihre Wahlbeteiligung etwa ein Fünftel unter dem Durchschnitt der Gesamtbevölkerung (Akarui senkyo suishin kyōkai 2016b). Um ein repräsentativeres Meinungsbild zu erlangen und damit eine ungleiche demokratische Repräsentation zu korrigieren, werden die Deliberationsteilnehmer per Zufall ausgewählt.

Drittens trägt der Mangel an parteipolitischen Alternativen zur Liberaldemokratischen Partei (LDP), die seit Jahrzehnten quasi das Regierungsmonopol besitzt, zur verbreiteten Politikverdrossenheit bei. Die Sozialistische Partei Japans als vormals stärkste Oppositionspartei glitt nach Ende des Kalten Kriegs in die politische Bedeutungslosigkeit ab. Angesichts ihres spektakulären Wahlsiegs 2009 galt die Demokratische Partei Japans (DPJ) als neue politische Alternative zur LDP. Die politischen Hoffnungen zerschlugen sich jedoch zusehends durch das unzulängliche Krisenmanagement der DPJ-Regierung nach der Dreifachkatastrophe von 2011. Das Fehlen einer starken parlamentarischen Opposition mindert die horizontale Gewaltenkontrolle. Demokratische Innovationen stärken indes die ebenfalls unzulängliche 
vertikale Kontrolle in der japanischen Demokratie, indem sie den Input von Bürgern bei politischen Entscheidungsprozessen fördern.

Viertens kamen die politisch Verantwortlichen nach dem Reaktorunfall ihrer Rechenschaftspflicht gegenüber der Öffentlichkeit über ihr Krisenmanagement nur unzulänglich nach. Infolge forderten Bürger von ihren Repräsentanten mehr politische Transparenz. Die japanische Politik wird indes traditionell als ein closed shop beschrieben, dessen Akteure sich aus einem kleinen Machtzirkel rekrutieren. Persönliche Netzwerke und die daraus resultierenden engen Allianzen zwischen Politikern, Bürokraten und der Industrie führten dazu, dass die politische Elite über Jahrzehnte die Interessen von Bürgern überging oder paternalistische Entscheidungen traf (Schmidt 2005, S. 64; Hasegawa 2018, S. 135). Als Mittel gegen die herkömmliche, schwer demokratisch kontrollierbare Entscheidungsweise fördern deliberative Innovationen die demokratische Kontrolle und Transparenz, indem sie den Bürgern Mitbestimmungschancen einräumen und dafür ausgewogene Informationen zu dem zu diskutierenden politischen Themabereitstellen.

Fünftens mobilisierten neue soziale Bewegungen die Bevölkerung nach dem Reaktorunfall in einem Ausmaß, das nur mit den Massendemonstrationen gegen die Revision des japanisch-US-amerikanischen Sicherheitsvertrags 1960 vergleichbar ist. Die jüngere Demonstrationswelle führte jedoch zu keiner gesamtgesellschaftlichen Politisierung, wenngleich bei Meinungsumfragen selbst sechs bzw. sieben Jahre nach dem Reaktorunfall zwischen 55 und $60 \%$ der Befragten für einen Atomausstieg plädieren (Mainichi shinbun 2017; Asahi shinbun 2018).

Sechstens wird die japanische Zivilgesellschaft in der Literatur hinsichtlich ihrer Interessenvertretung gegenüber der institutionalisierten Politik und damit hinsichtlich ihres politischen Einflusses als schwach beschrieben (Pekkanen 2006, S. 7; Avenell 2018, S. 19-20; Vinken et al. 2010, S. 7; Yamamoto 2017, S. 51-52; Hasegawa 2018, S. 119). Politische Aktivitäten von zivilgesellschaftlichen Akteuren in der Öffentlichkeit tragen dazu bei, die repräsentative Demokratie lebendig auszugestalten. Die Zivilgesellschaft bietet dabei einen Raum für einen freien Meinungsaustausch ohne staatliche Kontrolle bzw. Einflussnahme, weshalb dort deliberative Demokratie gelebt wird (Tamura 2017, S. 36).

\section{Hauptcharakteristika von lokalen Planungszellen und der nationalen Deliberative Poll}

Deliberation wird hier als freie Gruppendiskussion zwischen Bürgern zu einem vorgegebenen politischen Thema definiert. Deliberative Verfahren werden eingesetzt, um dem Gemeinsinn entsprechende, konsensorientierte Politikansätze zu erarbeiten. Sie sollen Prozesse des thematischen Erkenntnisgewinns, demokratischen Lernens und gleichberechtigten Meinungsaustausches sowie der rationalen Meinungsbildung in Gang setzen, wobei die einzelnen Ziele zeitlich ineinandergreifen. (Abb. 1).

Die hier ausgewählten Deliberationsbeispiele weisen methodische Gemeinsamkeiten (Zufallsauswahl, Informationsbereitstellung, Moderation, konsultative Bürgerempfehlung) auf: Durch die Zufallsauswahl sollen die Diskussionsgruppen die gesamtgesellschaftliche Sozialstruktur annähernd abbilden. Da in einer repräsen- 


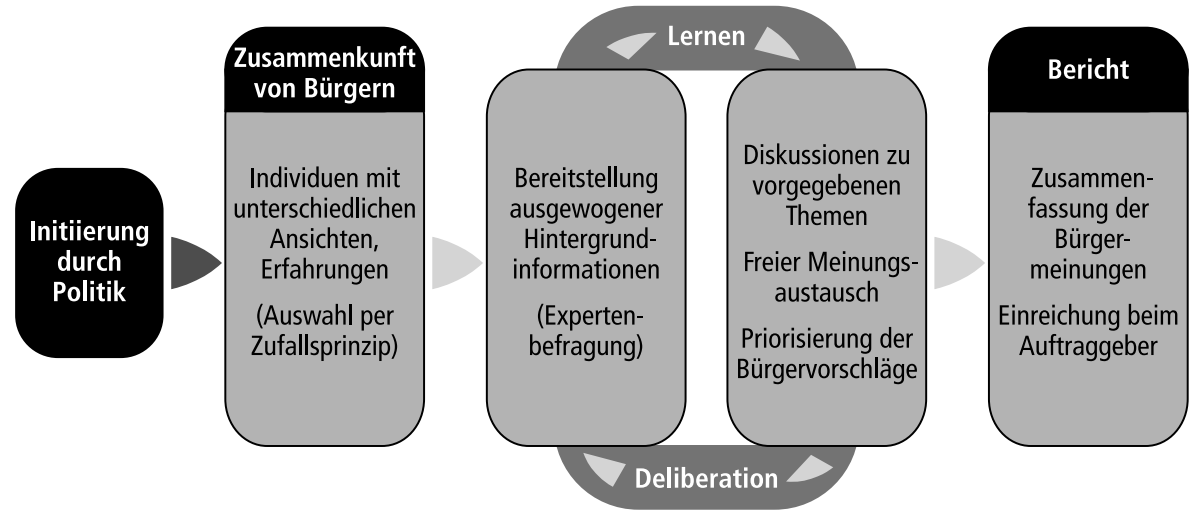

Abb. 1 Deliberations Konzept. (Eigene Darstellung)

tativen Demokratie nicht alle Bürger alles mitgestalten können, erhält eine kleine repräsentative Gruppe die Chance zur Mitbestimmung (Steiner 2012, S. 9). Zweitens bereiten die Organisatoren die Informationen mit dem Anspruch der thematischen Ausgewogenheit auf und stellen sie den Teilnehmern als Diskussionsgrundlage zur Verfügung. Da die Deliberationen in einem machtfreien Raum stattfinden sollen, intervenieren die organisierenden Institutionen nicht. Als dritte methodische Gemeinsamkeit begleiten vielmehr unabhängige Moderatoren die Gruppendiskussionen. Eine Moderation durch Dritte soll allen Meinungen und Interessen das gleiche Gewicht einräumen. Die von den Teilnehmern erarbeiteten Vorschläge haben viertens für die institutionellen Entscheidungsträger keinen bindenden, sondern nur einen konsultativen Charakter (Shinohara 2012, S. 242; Fishkin 2012, S. 74-75; Hendriks 2005, S. 84-85, 90, 96; Landwehr 2012, S. 360, 336).

Der Wuppertaler Soziologe Peter Dienel konzipierte in den 1970er Jahren die Planungszellen als deliberative Methode. Geläufiger als eine direkte japanische Übersetzung des ursprünglichen Begriffs „Planungszelle“ (keikaku saibō) ist in der japanischen Fachliteratur der Terminus „Deliberatives Bürgerforum“ (shimin tōgikai), der einen präziseren Eindruck von der Methodik vermittelt. Nachdem die Stadt Mitaka (Präfektur Tokyo) das Deliberationsverfahren als erste japanische Kommune in 2006 umsetzte, fand es in vielfältiger Umsetzung lokale Nachahmung. Auch andere Gemeinden setzten die Methode insbesondere in den Politikfeldern der Stadtgestaltung und Sozialpolitik ein (Shinoto 2005, S. 126, 2014, S. 214, 224). Dabei weisen die japanischen Planungszellen trotz ihrer lokalspezifischen Umsetzungen folgende Gemeinsamkeiten auf:

- Workshop über $1 \frac{1}{2}-2$ Tage

- Zufallsprinzip für die Auswahl von 20-60 Teilnehmern

- Diskussionsgruppe von 5-6 Personen

- Finanzielle Entschädigung für die Teilnahme

- Ausgewogene Informationen vorab

- Neutralität: keine Intervention der Exekutive, unabhängige Diskussionsmoderation

- Konsultativer Abschlussbericht 
Die japanische Variante der Bürgerworkshops fällt kürzer und kleiner aus als die zwei- bis viertägigen Planungszellen mit insgesamt 100-300 Bürgern in Deutschland. Der Rastlosigkeit der japanischen Gesellschaft geschuldet, beschränkt sich die Dauer der japanischen Planungszellen auf ein Wochenende. Dabei liegt die Teilnehmerzahl mit weniger als 100 Bewohnern auch unter dem ursprünglichen Modell. Die japanische ,light-Version“ hat indes den Vorzug, weniger Kosten zu verursachen (Tsubogō 2015, S. 172; Shinoto 2014, S. 218).

Als zweite Deliberationsmethode wird die Deliberative Poll (tōrongata yoron chōsa) untersucht. James Fishkin von der Stanford University entwickelte sie mit dem Ziel, dass sich Bürger unter idealen Umständen eine informierte Meinung bezüglich eines vorgegebenen Politikthemas bilden können (Fishkin 2012, S. 76). Dafür werden Methoden der Meinungsumfrage (basierend auf Mehrheitsentscheidungen) und Deliberation (basierend auf Konsensbildung) kombiniert. Es soll während des Experiments ermittelt werden, wie ein repräsentatives Gesellschaftssample seine Einstellungen zu einem vorgegebenen Thema ändert. Dafür werden Fragebögen eingesetzt, welche die Teilnehmer am Anfang und Ende einer Deliberative Poll beantworten. Zunächst werden Bürger per Zufallsauswahl in einer Telefonumfrage nach ihrer Meinung befragt. Die Organisatoren rekrutieren aus diesem statistisch repräsentativen Pool 200-600 Befragte für den eigentlichen Deliberationsworkshop. Ferner stellen sie ausgewogene Informationen nicht nur für die Gruppendiskussionen, sondern auch für die vorgeschalteten öffentlichen Anhörungen (public hearings) und Stellungnahmen (public comments) zur Verfügung. Während des Workshops besteht für die Teilnehmer die Möglichkeit, Experten zu befragen. Unabhängige, speziell geschulte Moderatoren begleiten die Gruppendiskussionen (Ladd 1996, S. 41; Participedia 2009; Fishkin 2012, S. 72).

In Japan fand die Deliberative Poll-Methode erstmals im Jahre 2012 auf nationaler Ebene Anwendung. Die DPJ zeigte sich der Bürgerpartizipation gegenüber aufgeschlossener als die LDP. Deshalb bot ihre kurze, die LDP-Vorherrschaft intermittierende Regierungsperiode ein günstiges Zeitfenster, um dieses dialogorientierte Partizipationsverfahren auch auf der nationalen Ebene zu implementieren. Das DPJKabinett beschloss 2011 unter dem Eindruck des Reaktorunfalls, die bisherige japanische Energiepolitik gänzlich auf den Prüfstand zu stellen. Die nationale Regierung wollte sich die gewichtige Entscheidung über ihre zukünftige Energiestrategie durch eine direkte Bürgerbeteiligung zusätzlich legitimieren lassen. Im Rahmen der Deliberative Poll standen der japanischen Gesellschaft drei Energieszenarien (bis zum Jahr 2030 0\%, $15 \%$ oder 20-25\% des Stroms aus AKWs zu speisen) zur öffentlichen Diskussion. Als Diskussionsgrundlage veröffentlichten die Organisatoren auf einer offiziellen Internetseite (Naikaku kanbō 2012a) die Risiken der einzelnen Energiestrategien wie das der globalen Erwärmung, Reaktor- oder Versorgungssicherheit (The Energy and Environment Council 2012). (Abb. 2).

Zahlreiche landesweite, öffentliche Diskussionsveranstaltungen mit Bürgern, Interessenvertretern und Experten gingen dem eigentlichen Deliberationsworkshop voraus. In den öffentlichen Anhörungen oder mittels der offiziellen Webseite konnten sich interessierte Bürger eine Meinung bilden oder öffentlich zu den Energiestrategien Stellung nehmen. Die eigentliche Deliberative Poll fand in Form der zweiwöchigen Telefonumfrage, an der 6849 Personen landesweit teilnahmen, und des zweitägi- 


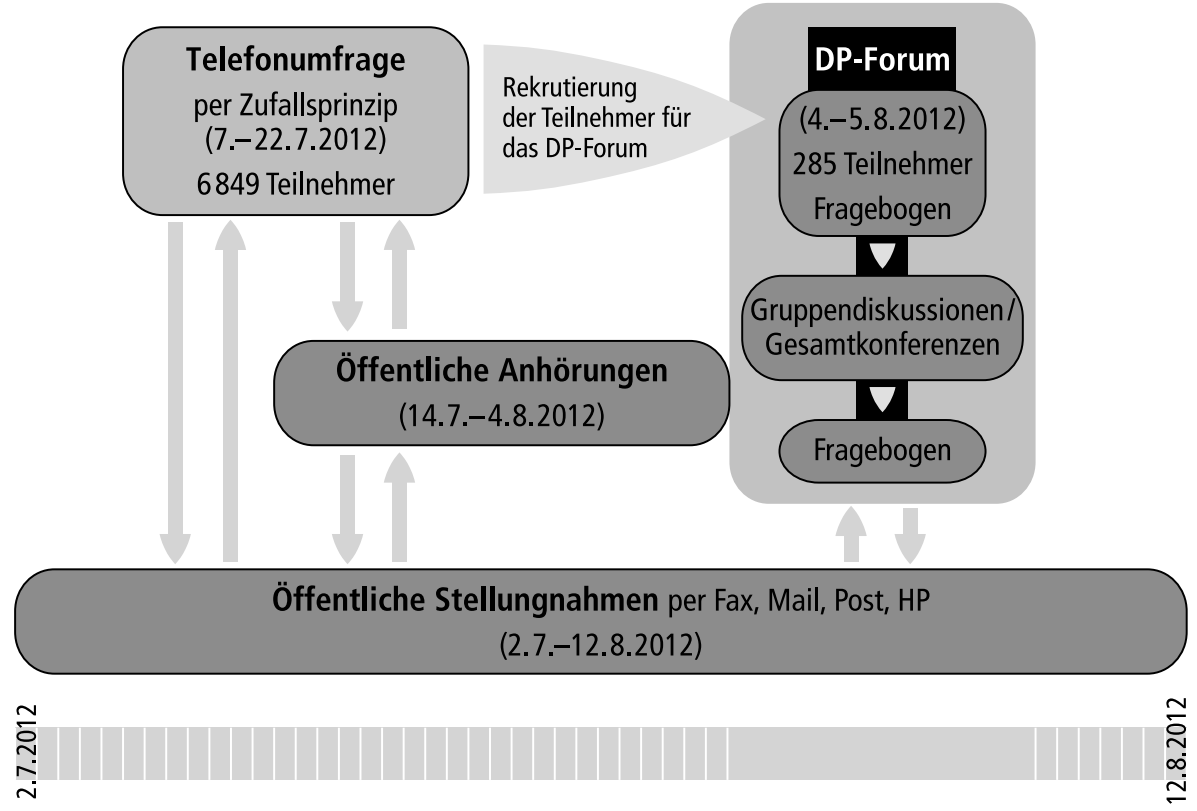

Abb. 2 Nationale Deliberative Poll. (Eigene Darstellung)

gen Workshops mit 285 Teilnehmern in Tokyo statt. Alle japanischen Atomreaktoren waren zum Zeitpunkt der öffentlichen Diskussionen im Sommer 2012 heruntergefahren (Paulitz 2015; World Nuclear Power Association 2018).

\section{Evaluationskriterien für die empirischen Deliberationsfälle}

Demokratiekonzepte erwarten von einer Ausweitung der politischen Partizipationschancen mittels demokratischer Innovationen, dass diese die Demokratiequalität verbessern. Deshalb wird hier auf Studien Bezug genommen, welche die Qualität verfasster Demokratien messen oder den Output demokratischer Innovationen bewerten. Erstere rekurrieren zumeist auf einem Kriterienkatalog von Larry Diamond und Leonardo Morlino. Anhand des empirisch konstatierten Ausmaßes von Partizipation, politischer Gleichheit, vertikaler Rechenschaftsplicht und Responsivität wird die demokratische Performanz von Staaten evaluiert (Campbell und Barth 2009; Pickel und Pickel 2006; Diamond und Morlino 2004, S. 22-23; Diamond und Morlino 2005). Die in der Literatur zu demokratischen Innovationen mehrheitlich genannten Evaluationskriterien (Input-Legitimität, demokratischer Prozess, politische Bildung und Effektivität), die hier für eine Bewertung der empirischen Deliberationsbeispiele zusätzlich hinzugezogen werden, korrespondieren mit den Demokratieindizes (Geißel 2008, S. 233-235; Geissel 2012, S. 167-170). Im Folgenden werden beide Kriterien miteinander verknüpft, um den Einfluss von deliberativen Innovationen auf die Qualität der repräsentativen Demokratie in Japan zu untersuchen. 
Partizipation (participation) bildet das erste Qualitätskriterium, da sie für eine verfasste Demokratie unabdingbar ist. Wieviel Gelegenheit den Bürgern zur Mitbestimmung eingeräumt wird und in welchem Maße sie damit Policies tatsächlich inhaltlich mitgestalten können, bestimmt die demokratische Qualität von Partizipationsverfahren. Demokratische Innovationen bieten über die herkömmlichen Gelegenheiten wie Wahlen hinaus neue Mitbestimmungsarenen. Indem Bürger an einer politischen Entscheidungsfindung partizipieren können, nehmen die Akzeptanz der daraus entwickelten Politiken und die sich daraus ableitende Akzeptanz der Verantwortlichen durch die Wähler zu (Input-Legitimität) (Geißel 2008, S. 233-234; Geissel 2012, S. 167, 170).

Ein gesellschaftlich ausgewogenes Sample von Teilnehmern fördert die Legitimität partizipativer Entscheidungen. Um eine sozial unausgewogene Interessenvertretung - wie häufig an direkten, frei zugänglichen Beteiligungsmethoden kritisiert zu vermeiden, rekrutieren die hier untersuchten Deliberationsbeispiele ihre Teilnehmer mit statistischen Auswahlverfahren. Die Zufallsauswahl trägt zur politischen Gleichheit (equality) der Partizipierenden als zweitem Kriterium der Demokratiequalität bei. Das erstellte Sample soll ein annähernd repräsentatives Abbild der Gesamtgesellschaft bieten. Zudem stellen strenge deliberative Verfahrensregeln, die einen freien Meinungsaustausch auf gleicher Augenhöhe ermöglichen, auch eine politische Gleichheit her.

Die Teilnehmer machen Partizipationserfahrungen, die ihre politische Kompetenz erweitern. Politische Bildung (civic education) als drittes Kriterium, demokratische Innovationen zu evaluieren, trägt auch, wenngleich mittelbar, zur politischen Gleichheit bei. Auf Grundlage der aufbereiteten Informationen sowie den deliberativen Gruppendiskussionen eignen sich die Teilnehmer politisches Wissen an, was ihrem Empowerment dient (Geißel 2008, S. 235, 242-243; Geissel 2012, S. 169).

In welchem Maße die konzeptionellen Deliberationsnormen umgesetzt werden, beeinflusst die Qualität des demokratischen Meinungsbildungsprozesses (democratic process). Von normativen Demokratietheorien geprägte Deliberationskonzepte sehen in der Deliberation die Ideale von liberalen Demokratien - wie Toleranz gegenüber Andersdenkenden sowie eine freie Meinungsbildung und -äußerung - verwirklicht. Die Umsetzung demokratischer Normen in Partizipationsverfahren dient als viertes Erfolgskriterium für demokratische Innovationen (Geißel 2008, S. 233-234; Geissel 2012, S. 167, 170; Fishkin 2012, S. 73).

Responsivität (responsiveness) stellt ein weiteres Merkmal für die demokratische Qualität dar. Sie drückt sich in der Bereitschaft der Repräsentanten aus, Wünsche, Interessen und Erwartungen der Repräsentierten aufzugreifen. Indem den Bürgern zusätzlich zu Wahlen weitere Partizipationsgelegenheiten eingeräumt werden, wird die institutionelle Politik an den Wählerwillen zusätzlich rückgekoppelt und damit legitimiert. Die Responsivität von Politikern zeigt sich ferner in einer effektiven Umsetzung der Bürgerinteressen.

In einer Demokratie sind die Repräsentanten zur Rechenschaftsplicht (accountability) als weiteres Qualitätsmerkmal verpflichtet. Dieses Instrument der Gewaltenkontrolle fordert von den Regierungs- und Parlamentsvertretern, ihr Handeln sowohl gegenüber den Wählern als auch gegenüber den anderen demokratischen Institutionen zu erklären. Kommen sie ihrer Verantwortung nicht nach, so können sie des Amts 
enthoben werden. Je größer die Bereitschaft der Repräsentanten ist, sich responsiv $\mathrm{zu}$ zeigen und Rechenschaft abzulegen, desto effektiver wird die Bürgermeinung politisch umgesetzt (Morlino 2011, S. 201).

Effektivität (effectiveness) ist schließlich als Evaluationskriterium für demokratische Innovationen relevant. Je kongruenter sich die Erwartungen der Stakeholder mit der politischen Umsetzung decken, umso effektiver sind die mittels deliberativer Verfahren getroffenen institutionellen Entscheidungen (Diamond und Morlino 2004, S. 23, 25; Campbell und Barth 2009, S. 212; Geißel 2008, S. 233-235; Geissel 2012, S. 167-170). Die genannten Kriterien werden in den folgenden beiden Kapiteln angewandt, um die Deliberationsbeispiele im Hinblick auf ihren Beitrag zur Demokratiequalität zu bewerten.

\section{Die Evaluation japanischer Planungszellen}

Nach den positiven Erfahrungen in der deutschen kommunalen Praxis erachteten japanische Gemeinden die Planungszellen als geeignetes Instrument, um die Mitbestimmung in der lokalen Selbstverwaltung zu erweitern. Aus der Vielzahl an kommunalen Implementationen wird hier am Beispiel der Stadt Mitaka der Beitrag deliberativer Innovationen zu einer Steigerung der Demokratiequalität untersucht. Als Vorreiter führte sie neun Planungszellen seit 2006 durch. Die Gemeindeverwaltung wählte die Teilnehmer aus dem Melderegister zufällig aus. Fünf Gruppen zu je fünf Teilnehmern erarbeiteten Vorschläge zur Stadtgestaltung.

Planungszellen institutionalisieren Beteiligungschancen und fördern damit die demokratischen Kompetenzen und Autonomie von Bürgern. Mit Planungszellen soll den Herausforderungen der japanischen Demokratie - wie dem geringe öffentliche Interesse an Politik - entgegengetreten werden. Die Teilnehmerzahlen sind allerdings im Fall Mitakas, wie bei japanischen Planungszellen gängig, auf insgesamt 25 Personen beschränkt. Somit bildete das Sample vom Jahr 2015 lediglich 0,018\% der Gesamtbevölkerungszahl von ca. 183.000 Einwohnern ab. Dies zog aus Wissenschaft und Praxis Kritik nach sich, dass das Sample nicht repräsentativ sei (Ayano 2014, S. 238; Shinoto 2014, S. 224). Aufgrund der minimalen Partizipationsrate können Planungszellen nur als eine Methode unter anderen gelten, welche die InputLegitimität als Qualitätskriterium für demokratische Innovationen steigert.

Die herkömmliche Partizipationspraxis von japanischen Gemeinden unterscheidet sich deutlich von der Zufallsauswahl der Planungszellen. Vormals verlas die Verwaltung die an kommunalen Entscheidungsprozessen teilnehmenden Bürger von Hand. Sie rekrutierte Ausschussmitglieder, die die Bürgerschaft vertreten sollten, zumeist aus Nachbarschaftsorganisationen. Deren aktive Mitglieder unterstützten in ihrem Quartier teilweise die kommunale Daseinsvorsorge, wie die Mülltrennung zu überwachen. Die Verwaltung konnte mit der Vergabe der Kommissionsitze an die Nachbarschaftsvertreter davon ausgehen, mit kooperationswilligen Bürgern zusammenarbeiten zu können. Mit der Partizipation von handverlesenen Bürgern verlieh die Gemeindeverwaltung ihrer häufig im Detail ausgearbeiteten Agenda einen partizipativ-demokratischen Anstrich (Shinoto 2014, S. 226; Hüstebeck 2014, S. 163-169). Indem hingegen die Zufallsauswahl allen gemeldeten Anwohnern die Chance bietet, 
an einer Planungszelle teilzunehmen, wird die politische Gleichheit als Demokratiekriterium gestärkt. Die Planungszellen sollen ein sozial ausgewogenes politisches Meinungsbild schaffen und damit partizipativen Herausforderungen wie dominanten Interessensgruppen und einem sozialen Bias begegnen.

Eine politische Gleichheit im Sinne eines ausgewogenen Verhältnisses zwischen den Geschlechtern und Alterskohorten konnte in Mitaka weitestgehend hergestellt werden (Shinoto 2014, S. 220-221). Ältere Männer sind wie bei anderen direkten Partizipationsformen überproportional vertreten. Da es den Angeschriebenen freisteht, dem Workshop fernzubleiben, folgen der Einladung weniger Berufstätige oder Bewohner, die in die familiäre Fürsorge eingebunden sind. Bei der Planungszelle 2018 waren dementsprechend 53\% der Teilnehmer im Vergleich zu 49\% der Gesamtbevölkerung männlich. Stärker fällt eine Überalterung ins Gewicht. Die Kohorte der über 50-Jährigen wurde in der Planungszelle durch $64 \%$ repräsentiert. In der Gesamtbevölkerung stellt sie aber lediglich $47 \%$ dar (Mitaka 2018, S. 51, 55).

Das Maß, indem die Deliberationsnormen in Form von demokratischen Idealen wie Gleichheit, Toleranz und Freiheit umgesetzt werden, bestimmt die Qualität des demokratischen Prozesses als Bewertungskriterium für deliberative Innovationen. Den Deliberationsnormen widerspricht es, wenn die Exekutive in den Meinungsbildungsprozess der Bürger eingreift. Die Diskussionsrunden werden deshalb nur an kritischen Punkten moderiert. Allerdings legt die Exekutive die von den Bürgern zu diskutierenden Themen fest, was den unabhängigen Charakter der Methode schmälert. Im Falle Mitakas organisiert und führt eine NPO die Planungszellen durch, was die Unabhängigkeit von der Gemeindeexekutive unterstreichen soll. De facto werden die Planungszellen jedoch per Vertrag delegiert und outgesourct.

Politische Bildung fördert die Qualität demokratischer Innovationen. Die Teilnahme an Planungszellen gilt wie andere Partizipationschancen als bereichernde individuelle Erfahrung, die demokratisches Lernen ermöglicht, d.h. die politischen Kenntnisse und Meinungsbildung der Teilnehmer vertieft und deren politisches Interesse sowie Bewusstsein stärkt (Hendriks 2005, S. 91; Sturm 2005, S. 41-42; Hungerland 2005, S. 31-32). Auch bei den Deliberationsprozessen in Mitaka analysiert Ayano Hirose (2014, S. 242-243), dass sich Planungszellenteilnehmer, die anfänglich nur zögerlich mitdiskutierten, im Verlauf reger beteiligten. Die Partizipation an der Mitakaer Planungszelle von 2015 motivierte laut eines Fragebogens, der am Ende verteilt wurde, 92,6\% der Befragten zu weiterem lokalen Engagement. Sie gaben mehrheitlich an, dass dadurch ihr Interesse an dem Thema Stadtgestaltung gestiegen $(56,8 \%$ ) oder sehr gestiegen sei (40,7\%) (Hüstebeck 2014, S. 183; Mitaka 2015, S. 82-83). Angesichts der „,sonst eher politikfeindlichen öffentlichen Kultur“ in Japan schätzt Shinoto Akinori (2014, S. 229) dieses demokratische Empowerment als bedeutend ein. Eine Förderung der politischen Bildung ist jedoch nur schwer zu evaluieren, da die Selbsteinschätzungen keine persönlichen Voraussetzungen wie politische Vorkenntnisse oder die soziale Stellung berücksichtigen.

Inwieweit die Interessen der Bürgerschaft politisch umgesetzt werden, verdeutlicht maßgeblich die Responsivität als weiteres Demokratiekriterium. Die interviewten Mitakaer Gemeindevertreter sahen trotz Zufallsauswahl das Problem, dass die Teilnehmer nicht durch ein politisches Mandat legitimiert seien. Letztendlich obliegt es den Repräsentanten, die Bürgerempfehlungen politisch umzusetzen. Responsivi- 
tät bedeutet deshalb nicht, die Verantwortung für politische Entscheidungen an die Repräsentierten zu delegieren. Die Exekutive muss die Bürgerempfehlungen auf ihre Praktikabilität und Finanzierbarkeit hin prüfen. In der Praxis implementiert sie deshalb i.d.R. nur einen Teil der Empfehlungen. Die bedingte Responsivität schmälert eine effektive Umsetzung des Bürgerberichts und damit den Implementationserfolg von demokratischen Innovationen (Hüstebeck 2014, S. 182-183; Hendriks 2005, S. 94).

Resümiert man die Stärken und Schwächen von Planungszellen, so liegt eine der Hauptstärken darin, dass Bürger - abgesehen von der Themenvorgabe - ohne institutionelle Auflagen kreativ Lösungsansätze entwickeln können. Dies ermöglicht einerseits innovative Politikvorschläge, andererseits erschwert diese Freiheit auch eine effektive Umsetzung. Der diskursive Meinungsbildungsprozess erweitert die demokratischen Kenntnisse und Tugenden der Teilnehmenden. Wie andere Deliberationsverfahren ist die Methode indes in der Planung und Umsetzung ressourcenintensiv, so dass die Verantwortlichen sie nur punktuell einsetzen.

\section{Die Evaluation der ersten nationalen Deliberative Poll}

Als weiteres Deliberationsbeispiel wird die nationale Deliberative Poll anhand der oben ausgeführten Kriterien evaluiert. Ziel der Deliberative Polling-Methode ist es, unter optimierten Rahmenbedingungen eine öffentliche Meinung herzustellen (Fishkin und Farrar 2005, S. 71). Inwiefern dieses Experiment einen partizipatorischen Impetus für ein Politikfeld mit weitreichenden Folgen für die Gesamtgesellschaft gab und welcher Beitrag damit zur Demokratiequalität geleistet wurde, wird im Folgenden analysiert.

Die Rechenschaftspflicht ist eines der Kriterien für die Qualität von repräsentativen Demokratien. Die DPJ-Regierung geriet nach der Dreifachkatastrophe in die Kritik, ungenügend die Umstände des Reaktorunfalls aufzuarbeiten und die Verantwortlichkeiten offenzulegen. Ministerpräsident Kan Naoto versprach als Reaktion auf den öffentlichen Druck, die bisherige nationale Energiepolitik zur Debatte zu stellen. Zur japanischen Deliberative Poll im Jahr 2012 diskutierten die Teilnehmer verschiedene Energieszenarien und stimmten unter dem Eindruck des Reaktorunfalls mehrheitlich für einen Atomausstieg. Die Schaffung dieser Partizipationsgelegenheit erfüllt das erste Kriterium für die Qualität von repräsentativen Demokratien.

Die politische Gleichheit als zweites Demokratiekriterium stützt sich auf die zufällige Teilnehmerauswahl der Deliberative Poll (Fishkin 2012, S. 76-78). Das mittels statistischer Verfahren gebildete Sample soll die Gesamtgesellschaft repräsentieren und damit die Input-Legitimität als Bewertungsmaßstab für demokratische Innovationen fördern. Eine bei der japanischen Deliberative Poll per Zufall ausgewählte Diskussionsgruppe schuf ein ausgewogeneres Meinungsbild als die frei zugänglichen Partizipationsforen, die den Workshop begleiteten. Das Votum für einen Atomausstieg zur Telefonbefragung mit $27 \%$ und am Workshopende mit $47 \%$ fiel deutlich geringer als das bei den vorgelagerten Veranstaltungen aus. Bei den landesweiten öffentlichen Anhörungen stimmten $68 \%$ und in den Stellungnahmen $87 \%$ für das $0 \%$-Szenario. Eine Meinungsäußerung per Fax, Brief oder Mail stand allen 
Bürgern offen, so dass sich überproportional Atomkraftgegner äußerten. Interessensvertreter der Anti-AKW-Bewegung hatten ihre Unterstützer z. B. durch Kommentarvorlagen mobilisieren können. Den landesweiten öffentlichen Anhörungen in 11 Städten ging eine öffentliche Ausschreibung voraus. Auch wenn die Teilnehmerzahl auf insgesamt 1542 Personen beschränkt wurde, bewarben sich überproportional viele Atomkraftgegner. Die Organisatoren waren sich bewusst, dass sich dadurch im Vergleich zu den statistisch basierten Meinungsumfragen mehr Personen äußerten, die sich bereits eine Meinung zum Energiethema gebildet hatten. Dass nicht die Regierung, sondern Kommunen, NGOs oder Universitäten die Interessenvertreter für die öffentlichen Anhörungen aussuchten, beeinflusste zusätzlich die hohe Zustimmung für einen Atomausstieg (Yanase 2015, S. 218, 276, 273; Naikaku kanbō 2012c). (Abb. 3).

Trotz der statistikbasierten Teilnehmerauswahl ist bei der japanischen Deliberative Poll ein im Vergleich zur Gesamtbevölkerung leichtes Ungleichgewicht bezüglich des Alters und Geschlechts zu erkennen. Wenngleich der Umfragepool statistisch repräsentativ gebildet wurde, so verzerrte sich das Sample durch die freiwillige Teilhabe. Teilnehmer, die 60 Jahre oder älter waren, bildeten dementsprechend mit $47 \%$ die größte Kohorte. Bei den unter 30-Jährigen ging die Schere zwischen dem Anteil an der Teilnehmergruppe $(15,4 \%)$ und an der Gesamtbevölkerung $(34,3 \%)$ am weitesten auseinander. Dass sich der Teilnehmerpool nur aus Nutzern einer Festnetznummer rekrutierte, erklärt u. a. die Diskrepanz. Jüngere wären eher über das Mobilfunknetz erreichbar gewesen. Die Durchführenden waren sich des methodisch bedingten Bias bewusst, aber zu dem Zeitpunkt war es rechtlich noch unzulässig, für politische Meinungsumfragen auf Handynummern zurückzugreifen.

Die freie Entscheidung des Einzelnen zu partizipieren - oder auch nicht -, trug ferner zur ungleichen Repräsentation bei. Die Alterskohorte, die mehrheitlich beruflichen und/oder familiären Verpflichtungen nachgeht, zeigte sich weniger zu einer Teilnahme bereit. Aufgrund des Aufwands, für den zweitägigen Workshop nach Tokyo zu kommen, waren auch Frauen mit $33 \%$ im Vergleich zu ihrem Anteil an der Gesamtbevölkerung (53,20\%) unterrepräsentiert (Naikaku kanbō 2012d, S. 2; Kokka senryaku tantō daijin 2012, S. 2; Naikaku kanbō 2012c; Yanase 2015, S. 276, 227; The Energy and Environment Council 2012). Die Abweichungen lassen den Schluss zu, dass diese sich auf das Endergebnis auswirkten. Der hohe Anteil an Alten förderte die Unterstützung des 0\%-Szenarios, da diese Kohorte die Kernenergie stärker als die nachfolgenden Generationen mit dem Schrecken und Leid der Atombombenabwürfe auf Hiroshima und Nagasaki assoziiert. Ein höherer Frauenanteil am Workshop hätte wahrscheinlich das 0\%-Szenario weiter gestützt, da sich

Abb. 3 Meinungsumschwung während des Deliberative Polling-Prozesses. (Eigene Darstellung)

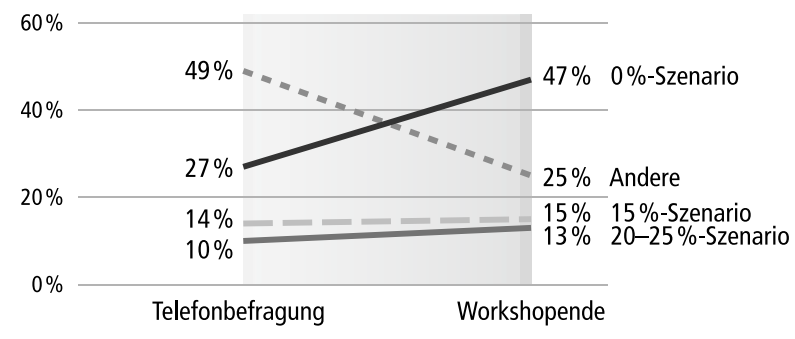


viele Mütter nach dem Reaktorunfall um die Gesundheit ihrer Kinder sorgten und deshalb die Kernenergie ablehnten. Die unzureichende Repräsentation von Jüngeren im Alter zwischen 20 bis 40 Jahren schmälerte hingegen eher das Votum für einen langfristigen Atomausstieg, da diese das Sicherheitsrisiko der AKWs bei Fragen nach Leitlinien für eine neue Energiestrategie geringer bewerteten.

Politische Gleichheit als Evaluationskriterium wird auch durch den demokratischen Prozess des strikt reglementierten Diskutierens hergestellt. Indem das Deliberative Polling demokratische Normen wie Toleranz und Gleichheit erfüllt, wird Demokratie er- und gelebt. Auch andere Deliberative Polls weisen soziale Unterschiede (wie bezügl. Bildung, Status oder Geschlecht) im Sample auf. Die daraus resultierende anfängliche Meinungsführerschaft einzelner Teilnehmer nimmt allerdings i.d.R bei zunehmender Diskussionserfahrung der anderen Beteiligten wie bei den Planungszellen ab (Fishkin 2012, S. 76-78). In der japanischen Teilnehmerbefragung gab mehr als die Hälfte an, dass das gegenseitige Verständnis für die Meinung des Anderen im Laufe der Diskussionen stieg. Die Moderation der Gruppendiskussion trug ferner dazu bei, dass die verschiedenen Beiträge als gleichwertig erachtet und gleichermaßen im Meinungsbildungsprozess berücksichtigt wurden. Darüber hinaus förderten die Mehrheitsentscheidungen mittels Fragebogen nach jeder Diskussionsrunde die politische Gleichheit (Yanase 2015, S. 204).

Unter den experimentellen Gegebenheiten einer Deliberative Poll weisen zahlreiche Fallstudien von der ersten Telefonbefragung bis zum Workshopende eine statistisch relevante Meinungsänderung auf (Fishkin und Farrar 2005, S. 79; Fishkin 2012, S. 73, 76; Luskin et al. 2007, S. 15). Den Wandel dokumentieren auch die japanischen Umfragen, die zu Beginn, nach jeder Diskussionsrunde und am Ende der Deliberative Poll ausgeteilt wurden. $27 \%$ der Teilnehmer befürworteten in der ersten Befragung das 0\%-Szenario. Die Zustimmung stieg am Ende auf $47 \%$ an. Das Votum für einen Atomausstieg von fast der Hälfte aller Teilnehmenden war ein für die Organisatoren unerwartet eindeutiges Ergebnis (Yanase 2015, S. 276; Naikaku kanbō 2012b).

Der Informations-/Erkenntnisgewinn der Teilnehmer unterstützte den Meinungswandel und förderte die politische Bildung als drittes Kriterium für demokratische Innovationen. Auch Analysen anderer Deliberative Polls zeigen, dass die bereitgestellten Informationen wesentlich zur politischen Gleichheit beitragen. Die Workshopteilnehmer investieren deutlich mehr Zeit in die Informationserfassung und Reflektion als diejenigen, die bei herkömmlichen Meinungsumfragen antworten. Ihr Wissensvorsprung gegenüber dem Bevölkerungsdurchschnitt verringert die Kluft zwischen politischen Laien und der gut informierten politischen Elite bzw. den Experten. Dabei sind die von den Organisatoren bereitgestellten Informationen nur eine Ressource unter anderen. Die meisten Teilnehmer nutzen i.d.R. 4-10 Wochen vor dem Workshop verstärkt Medien als weitere Informationsquelle und diskutieren das Thema in ihrem sozialen Umfeld (Fishkin und Farrar 2005, S. 79; Participedia 2009; Fishkin und Luskin 1996, S. 48-49).

Auch die japanischen Organisatoren stellten der Öffentlichkeit mittels ihrer Website und den landesweiten Informationsveranstaltungen zahlreiche Berichte zu den einzelnen Energieszenarien zur Verfügung. Zudem nahmen die Massenmedien das Thema auf und begleiteten den Deliberationsprozess, indem sie nicht nur über die 
Veranstaltungen berichteten, sondern auch eigene Meinungsumfragen zu den Energieszenarien veröffentlichten. So stand den Teilnehmern eine gut aufbereitete Informationsbasis für die Gruppendiskussionen zur Verfügung. Diese Informiertheit befähigte die Laien, die einzelnen Energiestrategien intensiv zu diskutieren und damit verbundene Risiken (Versorgungssicherheit, Klimabilanz etc.) zu erwägen. So drückt der Meinungswandel eine profunde thematische Auseinandersetzung und komplexe Meinungsfindung aus, die weit über ein auf „Ja-oder-Nein“ komprimiertes direktdemokratisches Votum zum Atomausstieg hinausgeht (Yanase 2015).

Das Maß, in dem die Repräsentanten auf das Endergebnis der Deliberative Poll politisch eingehen, offenbart deren Responsivität als viertes Kriterium für die Demokratiequalität. Der politische Nutzen stellt für die Repräsentanten den Anreiz dar, eine deliberative Entscheidung umzusetzen. Von der Deliberative Poll versprachen sich die politisch Verantwortlichen, eine gesellschaftsrelevante Policy angesichts des öffentlichen Drucks nach der Reaktorkatastrophe zusätzlich legitimieren zu können. Nach einer Kabinettsumbildung, wenngleich unter Führung der DPJ, zeigte sich die Regierung gegenüber dem abschließenden Votum von $47 \%$ für das $0 \%$-Szenario wenig responsiv, da parteiintern die Meinungen über eine zukünftige Atompolitik auseinandergingen. Trotz der hohen Zustimmung verschob die Regierung von Ministerpräsident Noda Yoshihiko den Atomausstieg um zehn Jahre, bis auf das Jahr 2040. Sein liberaldemokratischer Nachfolger, Abe Shinzō, überging nach seiner Amtsübernahme vollends das Ergebnis der Deliberative Poll. Bereits während des Wahlkampfs im Dezember 2012 hatte Abe einen ,sicheren“ Wiedereinstieg in die Atomenergie propagiert. Unter seiner Regierung gingen folglich bis Juli 2019 neun AKWs in Betrieb (Paulitz 2015; World Nuclear Power Association 2018; Nippon.com 2019). Die Gestaltung der zukünftigen Energiepolitik wurde mit der wiederhergestellten LDP-Dominanz unter Ausschluss der Zivilgesellschaft erneut zur Regierungssache gemacht. Damit wurde die aus der Deliberative Poll hervorgegangene öffentliche Meinung im Ergebnis weder responsiv von den politischen Repräsentanten aufgenommen, noch effektiv politisch umgesetzt.

In einer allgemeinen Beurteilung fällt auch Effizienz als eine weitere Schwachstelle von Deliberative Polls ins Gewicht. Die Kosten für die Durchführung sind selbst im Vergleich zu anderen deliberativen Verfahren hoch. Die Organisation, Rekrutierung und Durchführung nicht nur des Workshops selbst, sondern auch der vorgeschalteten öffentlichen Veranstaltungen erfordern erhebliche öffentliche Ressourcen. Dennoch ist in summa gerade wegen des hohen Verfahrensaufwands der Impact auf die öffentliche Meinung durch die mediale Berichterstattung etc. und damit die politische Wirkung ausgesprochen groß.

\section{Fazit}

Nach der eingehenden Analyse der Stärken und Schwächen von Planungszellen und einer Deliberative Poll ist zusammenfassend zu konstatieren, dass deliberative Verfahren die demokratische Qualität der japanischen Demokratie lediglich punktuell verbessern. Sie leisten einen demokratischen Input und erweitern das politische Wissen und die demokratischen Kompetenzen der Teilnehmer. Beide deliberative Ver- 
fahren wirken somit dem vorherrschenden politischen Desinteresse auf individueller Ebene entgegen. Den Beispielen sind hingegen auch eine geringe Effektivität und Responsivität gemein. Dies, obwohl die Deliberationen während einer frühen Phase der Politikgestaltung, in der noch politischer Gestaltungsspielraum besteht, implementiert werden. Dass das Energiethema polarisierte und die öffentliche Meinung dazu auseinanderging, unterscheidet die Deliberative Poll jedoch von den Planungszellen. In Japan werden Planungszellen vornehmlich angeboten, um Bürger in die langfristige Stadtplanung einzubeziehen. Die Praxis in anderen Ländern zeigt indes, dass die Deliberationsmethode durchaus auch auf konfliktreichere Themen anwendbar ist. Dies würde der normativ angestrebten Konsensfindung von Deliberationen näherkommen.

Auch die enge Thementaktung auf $90 \mathrm{~min}$ pro Thema wird dem konzeptionellen Anspruch eines tatsächlichen Meinungsaustausches und einer Konsensfindung nicht gerecht. Die Verkürzung nimmt den Teilnehmern die Möglichkeit, das Thema ausführlich zu diskutieren, ihre Meinung dazu zu überdenken sowie schließlich eine gemeinsame Lösung zu finden. Bei der dichten Taktung besteht die Gefahr, dass Bürger nur einzelne Stellungnahmen abgeben, die der Politik lediglich als Alibi dienen. Im Artikel wurden weitere Grenzen von deliberativen Innovationen wie insbesondere die rechtlich nicht bindenden Bürgerempfehlungen offengelegt. Die Deliberationen schaffen ferner lediglich die Möglichkeit zur Teilhabe; sie mobilisieren jedoch nicht. Hybride Formen, die verschiedene innovative Partizipationsverfahren kombinieren, können Schwachstellen dieser Art kompensieren. Deliberative Polls z. B. vereinen bereits die methodischen Ansätze der Meinungsumfrage und Deliberation. Es ist des Weiteren denkbar, Deliberationen auch in Japan wie bereits in anderen Ländern mit anderen demokratischen Verfahren zu kombinieren und gezielt vor Referenden oder Wahlen zu schalten.

Deliberative Innovationen bieten nicht den ,großen Wurf“, um die Malaise der verfassten Demokratie in ihrer Gesamtheit zu kurieren. Indes verbessern Deliberationen auf der Mikroebene Teilaspekte der demokratischen Qualität, indem sie den japanischen Herausforderungen der Demokratie begegnen:

Vertrauenszuwachs in die politische Elite: Deliberationen wirken vor allem im interpersonalen Kontext, um Vertrauen in die politischen Institutionen zu fördern. Der persönliche Meinungs- und Informationsaustausch zwischen Exekutive und Bürgern schafft eine Vertrauensbasis, aus dem häufig weiteres Bürgerengagement hervorgeht. Die Teilnehmer an den untersuchten Planungszellen äußerten sich mit deutlicher Mehrheit positiv über ihre Deliberationserfahrungen. Indes ist die Teilnehmerzahl mit weniger als $1 \%$ der Gesamtbevölkerung so verschwindend gering, dass die Erfahrung von Einzelnen nicht zu einem gesamtgesellschaftlichen Vertrauenszuwachs in die politische Elite und Institutionen führen könnte.

Beteiligung insbesondere junger Wähler: Trotz der positiven individuellen Partizipationserfahrungen der Deliberationsteilnehmer herrscht in der japanischen Gesamtgesellschaft politisches Desinteresse vor, das sich in einem Negativtrend bei der Wahlenthaltung ausdrückt. Die Zufallsauswahl der Deliberationsteilnehmer kann die geringe Wahlbeteiligung von insbesondere jungen Bürgern korrigieren, jedoch aufgrund der freiwilligen Teilnahme nicht ganz kompensieren. 
Responsivere, transparentere Politik: Demokratische Innovationen haben die Kultur der japanischen Politik verändert, die traditionell auf persönlichen, geschlossenen Netzwerken fußt. Die erfolgreich implementierten demokratischen Innovationen, insbesondere in der kommunalen Praxis, minderten weitverbreitete Vorbehalte der politischen Elite gegenüber partizipativen Verfahren. Die politisch Verantwortlichen gehen responsiver auf die Forderung nach mehr Bürgerbeteiligung ein und öffnen ihre Entscheidungsverfahren. Die institutionalisierten Partizipationsmethoden bieten der Öffentlichkeit einen Raum für eine politische Meinungsfindung. Der von deliberativen Verfahren angestoßene öffentliche Diskurs, die bereitgestellten Informationen sowie die Dokumentation gestalten den Policy-Prozess deutlich transparenter als die herkömmliche Entscheidungspraxis.

Größerer politischer Einfluss für die Zivilgesellschaft: Die wachsende Zahl an Partizipationsforen erweitert die politische Interessenvertretung zivilgesellschaftlicher Gruppen, obwohl ihr politischer Einfluss nach wie vor als gering erachtet wird. Auch wenn das Zufallsverfahren den organisierten Interessensgruppen den Zugang zum Workshop verstellte, gewährte ihnen das Deliberative Polling alternative demokratische Arenen. Die außerordentlich hohe Zustimmung für einen Atomausstieg in den öffentlichen Anhörungen und Stellungnahmen verdeutlicht, dass die zivilgesellschaftlichen Akteure ihre Strategie an die neuen Gegebenheiten angepasst haben und die neuen Foren nun auch für sich nutzen. Demokratische Innovationen wie diese erweitern langfristig das zivilgesellschaftliche Teilhaberepertoire und tragen zum öffentlichen politischen Diskurs bei, der die Demokratie stärkt.

Dass Deliberationen in der hier untersuchten institutionalisierten Form kein Mittel sind, um eine radikale Direktdemokratie zu verwirklichen, verdeutlichen die empirischen Fallbeispiele. Der nicht bindende, konsultative Charakter der Bürgerempfehlungen schiebt dem einen Riegel vor. Die politische Gestaltungsmacht bleibt in der Hand der politischen Institutionen. Sie behalten sich sowohl die Themensetzung als auch die Verfahrensorganisation und -durchführung vor. Die Kontrolle über die Themen- und Teilnehmerauswahl sowie den Outcome, die der Staat dabei behält, ist für die japanische Bürgerbeteiligung charakteristisch. In einem nach wie vor paternalistisch angelegten Bürger-Staat-Verhältnis misstrauen die politisch Verantwortlichen mehrheitlich der freien Meinungsäußerung und Interessenvertretung durch zivilgesellschaftliche Akteure. Die mangelnde politische Responsivität lässt erwarten, dass zumindest auf der nationalen Ebene keine weiteren Deliberationsverfahren in absehbarer Zeit umgesetzt werden.

Da die Vorbereitung, Rekrutierung und Implementation erhebliche öffentliche Ressourcen erfordern, bleiben Deliberationen in der realen Demokratie unabhängig von Partizipationsvorbehalten wie in Japan auch in anderen Staaten Ausnahmefälle. Die politisch Verantwortlichen wägen genau $a b$, ob sie diese Ressourcen für etwas mehr Input-Legitimität aufwenden. In der Regel bleibt die Legitimation auf eine periodische Beteiligung an Wahlen beschränkt. Die Kosten und analysierten Grenzen deliberativer Verfahren sprechen trotz der aufgezeigten positiven Demokratiebeiträge wie Transparenz oder Bürgerempowerment dafür, Deliberationen nur als ein Partizipationsinstrument unter anderen gegen die Krise der repräsentativen Demokratie einzusetzen. 
Danksagung Für ihre wertvollen Kommentare danke ich Patrick Köllner und Hannes Mosler sowie den beiden anonymen Gutachtern. Der Artikel entstand im Rahmen des vom Bundesministerium für Bildung und Forschung geförderten Projekts ,Deliberation als innovative Form von demokratischer Bürgerbeteiligung im japanisch-deutschen Vergleich“ (FKZ: 01UL1828X).

Open Access Dieser Artikel wird unter der Creative Commons Namensnennung 4.0 International Lizenz (http://creativecommons.org/licenses/by/4.0/deed.de) veröffentlicht, welche die Nutzung, Vervielfältigung, Bearbeitung, Verbreitung und Wiedergabe in jeglichem Medium und Format erlaubt, sofern Sie den/die ursprünglichen Autor(en) und die Quelle ordnungsgemäß nennen, einen Link zur Creative Commons Lizenz beifügen und angeben, ob Änderungen vorgenommen wurden.

\section{Literatur}

Akarui senkyo suishin kyōkai. 2016a. Sangiiin senkyo nendaibetsu tōhyōritsu no sū̄ (Wandel der Wahlbeteiligung nach Alterskohorte). http://www.akaruisenkyo.or.jp/070various/071syugi/693/. Zugegriffen: 15. Juli 2019.

Akarui senkyo suishin kyōkai. 2016b. Sangiiin senkyo tōhyōritsu no sū̄ (Wandel der Wahlbeteiligung). http://www.akaruisenkyo.or.jp/070various/071syugi/. Zugegriffen: 15. Juli 2019.

Asahi shinbun. 2018. Sagawa-shi no kokkai shochi hitsuyō 67\%. Asahi shinbun yoron chōsa (67\% halten eine Anhörung von Sagawa im Parlament für notwendig. Meinungsumfrage der Asahi shinbun). https://www.asahi.com/articles/ASL2M3GTPL2MUZPS001.html. Zugegriffen: 25. Juli 2019.

Avenell, Simon. 2018. Japan. In Routledge Handbook of civil society in Asia, Hrsg. Akihiro Ogawa, 17-32. London, New York: Routledge.

Ayano, Hirose. 2014. Die wissensgenerierende Stadt: Die Fallstudie der Bürgerdiskussionen in der Stadt Mitaka. In Die Qualität von Bürgerbeteiligungsverfahren. Evaluation und Sicherung von Standards am Beispiel von Planungszellen und Bürgergutachten, Hrsg. Hans-Liudger Dienel, 235-253. München: Oekom.

Campbell, David F.J., und Thorsten D. Barth. 2009. Wie können Demokratie und Demokratiequalität gemessen werden? Modelle, Demokratie-Indices und Länderbeispiele im globalen Vergleich. SWSRundschau 49(2):209-233.

Diamond, Larry Jay, und Leonardo Morlino. 2004. The quality of democracy: An overview. Journal of Democracy 15(4):20-31.

Diamond, Larry Jay, und Leonardo Morlino. 2005. Assessing the quality of democracy. Baltimore: John Hopkins University Press.

Fishkin, James. 2012. Deliberative polling: Reflections on an ideal made practical. In Evaluating democratic innovations. Curing the democratic malaise?, Hrsg. Brigitte Geissel, Kenneth Newton, 71-89. London, New York: Routledge.

Fishkin, James, und Cynthia Farrar. 2005. Deliberative polling: From experiment to community resource. In The deliberative democracy handbook. Strategies for effective civic engagement in the twenty-first century, Hrsg. John Gastil, Peter Levine, 68-79. San Francisco: Jossey-Bass.

Fishkin, James, und Robert C. Luskin. 1996. The deliberative poll: A reply to our critics. The Public Perspective 7:45-49.

Geißel, Brigitte. 2008. Zur Evaluation demokratischer Innovationen - die lokale Ebene. In Lokale Politikforschung heute, Hrsg. Hubert Heinelt, Angelika Vetter, 227-248. Wiesbaden: VS.

Geissel, Brigitte. 2012. Impacts of democratic innovations in Europe: Findings and desiderata. In Evaluating democratic innovations. Curing the democratic malaise?, Hrsg. Brigitte Geissel, Kenneth Newton, 163-183. London, New York: Routledge.

Geissel, Brigitte, und Kenneth Newton (Hrsg.). 2012. Evaluating democratic innovations. Curing the democratic malaise? London, New York: Routledge.

Hasegawa, Kōichi. 2018. Continuities and discontinuities of Japan's political activism before and after the Fukushima disaster. In Social movements and political activism in contemporary Japan, Hrsg. David Chiavacci, Julia Obinger, 115-136. New York: Routledge.

Hendriks, Carolyn M. 2005. Lay citizen deliberations: Consensus conferences and planning cells. In The deliberative democracy handbook. Strategies for effective civic engagement in the twenty-first century, Hrsg. John Gastil, Peter Levine. San Francisco: Jossey-Bass.

Hungerland, Beatrice. 2005. Bürgergutachten durch Planungszellen. In Die Befreiung der Politik, Hrsg. Peter C. Dienel, 30-34. Wiesbaden: VS. 
Hüstebeck, Momoyo. 2014. Dezentralisierung in Japan: Politische Autonomie und Partizipation auf Gemeindeebene. Wiesbaden: Springer VS.

Kokka senryaku tantō daijin. 2012. Senryaku sakutei ni mukete. Kokuminteki giron ga mezashi arawasu mono (Angesichts einer Strategieentwicklung, die den öffentlichen Diskurs widerspiegeln soll). https://www.cas.go.jp/jp/seisaku/npu/policy09/pdf/20120904/shiryo1-1.pdf. Zugegriffen: 30. Mai 2018.

Ladd, Everett C. 1996. Fishkin's "Deliberative Poll" is flawed science and dubious democracy. The Public Perspective 7(1):41-45.

Landwehr, Claudia. 2012. Demokratische Legitimation durch rationale Kommunikation. In Normative Demokratietheorien: Zeitgenössische Demokratietheorie, Bd. 1, Hrsg. Oliver W. Lembcke, Claudia Ritzi, und Gary S. Schaal, 355-385. Wiesbaden: VS.

Luskin, Robert C., James S. Fishkin, und Kyu S. Hahn. 2007. Deliberation and net attitude change. Paper Presented at the ECPR Conference, Pisa.

Mainichi shinbun. 2017. Honsha yoron chōsa. Genpatsu saikadō. Hantai 55\% sansei 26\%. Sa kakudai (Meinungsumfrage. 55\% gegen und 26\% für eine Wiederinbetriebnahme der Atomkraftwerke. Unterschied hat sich vergrößert). https://mainichi.jp/articles/20170313/k00/00m/010/101000c. Zugegriffen: 12. Juli 2019.

Mitaka. 2015. Dai 4ji kihon keikaku dai ichiji kaitei ni muketa 'Mitaka machizukuri desukasshon' jisshi hōkokusho (Bericht zur Umsetzung der „Mitakaer Stadtgestaltungsdiskussion“ über die erste Revision des 4. Mitakaer Grundlagenplans). Mitaka: Eigenverlag.

Mitaka. 2018. 'Mitaka-shi chōsha, gijō tō tatekae kihon kōzō' sakutei ni muketa 'Mitaka machizukuri desukasshon' jisshi hōkokusho (Bericht zur Umsetzung der „Mitakaer Stadtgestaltungsdiskussion“ über eine Grunderneuerung der Bauten der Gemeindeverwaltung und des Rats). Mitaka: Eigenverlag.

Morlino, Leonardo. 2011. Changes for democracy: Actors, structures, processes. Oxford: Oxford University Press.

Naikaku kanbō. 2012a. Hanasō eneruḡ̄ to kankyō no mirai (Lasst uns über die Zukunft der Energie und Umwelt reden). https://www.cas.go.jp/jp/seisaku/npu/policy09/sentakushi/index.html. Zugegriffen: 28. Juni 2019.

Naikaku kanbō. 2012b. Kaku chōsa no tokuchō to chōsa kekka ni tsuite (Über die Besonderheiten jeder Untersuchung und die Befragungsergebnisse). https://www.cas.go.jp/jp/seisaku/npu/policy09/ pdf/20120827/shiryo1.pdf. Zugegriffen: 30. Mai 2018.

Naikaku kanbō. 2012c. Senryaku sakutei ni mukete: Kokuminteki giron ga mezashi arawasu mono (Angesichts eines Strategieentwurfs, der die öffentliche Meinung widerspiegeln soll). https://www.cas.go. jp/jp/seisaku/npu/policy09/pdf/20120828/shiryo1-2.pdf. Zugegriffen: 30. Mai 2019.

Naikaku kanbō. 2012d. Tōrongata yoronchōsa no kekka ni tsuite (Die Ergebnisse der Deliberative Poll). https://www.cas.go.jp/jp/seisaku/npu/policy09/pdf/20120827/shiryo2-3-1. Zugegriffen: 30. Mai 2018.

Newton, Ken. 2012. Curing the democratic malaise with democratic innovations. In Evaluating democratic innovations. Curing the democratic malaise?, Hrsg. Brigitte Geissel, Kenneth Newton, 3-20. London, New York: Routledge.

Nippon.com. 2019. Japan's nuclear power plants. https://www.nippon.com/en/features/h00238/japan\%e2 \%80\%99s-nuclear-power-plants.html?cx_recs_click=true. Zugegriffen: 25. Juli 2019.

Offe, Claus. 2003. Demokratisierung der Demokratie: Diagnosen und Reformvorschläge. In Demokratisierung der Demokratie. Diagnosen und Reformvorschläge, Hrsg. Claus Offe, 9-24. Frankfurt am Main: Campus.

Participedia. 2009. Deliberative Polling. https://participedia.net/en/methods/deliberative-polling\#cite_ note-twenty_eight-27. Zugegriffen: 01. Februar 2019.

Paulitz, Henrik. 2015. Ringen um die Atompolitik in Japan. http://www.ippnw.de/commonFiles/pdfs/Info/ Ringen_um_die_Atompolitik_in_Japan.pdf. Zugegriffen: 30. Mai 2019.

Pekkanen, Robert. 2006. Japan's dual civil society: Members without advocates. Stanford: Stanford University Press.

Pickel, Gert, und Susanne Pickel (Hrsg.). 2006. Demokratisierung im internationalen Vergleich: Neue Erkenntnisse und Perspektiven. Wiesbaden: VS.

Saward, Michael. 2000. Democratic innovation: Deliberation, representation and association. London: Routledge.

Schmidt, Carmen. 2005. Japan's circle of power: Legitimacy and integration of a national elite. ASIEN 96:46-67.

Shinohara, Hajime. 2012. Shūshō. Jakkan no rironteki kōsatsu (Epilog. Einige theoretische Reflektionen). In Tōgi no demokurashī no chōsen: Mini paburikkusu ga hiraku atarashī seiji (Deliberative Demokra- 
tie wagen. Mini-publics eröffnen Chancen für eine neue Politik), Hrsg. Hajime Shinohara, 233-260. Tokyo: Iwanami.

Shinoto, Akinori. 2005. Die Planungszelle in Japan. In Die Befreiung der Politik, Hrsg. Peter C. Dienel, 125-127. Wiesbaden: VS.

Shinoto, Akinori. 2014. Deliberatives Bürgerforum in Japan: Ein Kompaktmodell? In Die Qualität von Bürgerbeteiligungsverfahren. Evaluation und Sicherung von Standards am Beispiel von Planungszellen und Bürgergutachten, Hrsg. Hans-Liudger Dienel, 213-233. München: Oekom.

Smith, Graham. 2009. Democratic innovations: Designing institutions for citizen participation. Cambridge: Cambridge University Press.

Steiner, Jürg. 2012. The foundations of deliberative democracy: Empirical research and normative implications. Cambridge: Cambridge University Press.

Sturm, Hilmar. 2005. Planungszellen-Teilnahme produziert Identität. In Die Befreiung der Politik, Hrsg. Peter C. Dienel, 40-44. Wiesbaden: VS.

Tamura, Tetsuki. 2017. Jukugi minshushugi-ron: Jukugi no ba to shite no shiminshakai-ron (Diskurs über deliberative Demokratie. Diskurs über die Zivilgesellschaft als Ort der Deliberation). In Shimin shakai-ron. Riron to jisshō no saizensen (Der Diskurs über die Zivilgesellschaft. An vorderster Front der Theorien und der Nachweise), Hrsg. Haruya Sakamoto, 20-38. Kyoto: Hōritsu bunkasha.

The Energy and Environment Council. 2012. Options for energy and the environment (Provisional translation). https://www.cas.go.jp/jp/seisaku/npu/policy09/sentakushi/pdf/Report_English.pdf. Zugegriffen: 30. Mai 2018.

Tsubogō, Minoru. 2015. Shimin sanka to gabanansu: Shimin no enpawāmento (Bürgerpartizipation und Governance. Bürgerempowerment). In Hikaku seijigaku no furontia. 21-seiki no seisaku kadai to atarashī rīdāshippu (Die Herausforderungen der vergleichenden Politikwissenschaft. Die Politikthemen des 21sten Jahrhunderts und die neue Führung), Hrsg. Norio Okazawa, 164-175. Kyoto: Mineruva shobō.

Vinken, Henk, Yuko Nishimura, und Bruce White. 2010. Introduction. In Civic engagement in contemporary Japan, Hrsg. Henk Vinken, Yuko Nishimura, Bruce L.J. White, und Masayuki Deguchi, 3-20. New York: Springer New York.

Watanabe, Masao, und Carmen Schmidt. 2013. Japans neuer Rechtspopulismus: Ursachen, Ziele und Perspektiven. Veröffentlichungen der Forschungsstelle Japan (23).

World Nuclear Power Association. 2018. Nuclear power in Japan. http://www.world-nuclear.org/ information-library/country-profiles/countries-g-n/japan-nuclear-power.aspx. Zugegriffen: 23. August 2018.

Yamamoto, Hidehiro. 2017. Shakai undō-ron: Kokka ni taikō suru shimin shakai (Theorien sozialer Bewegungen. Die Bürgergesellschaft, die dem Staat widersteht). In Shimin shakai-ron. Riron to jisshō no saizensen (Der Diskurs über die Zivilgesellschaft. An vorderster Front der Theorien und Nachweise), Hrsg. Haruya Sakamoto, 39-54. Kyōto: Hōritsu bunkasha.

Yanase, Noboru. 2015. Jukugi to tōgi no minshushugi-ron: Chokusetsu minshusei no minshushugi-ron (Deliberative und diskursive Demokratie. Ein direktdemokratischer Diskurs). Tokyo: Mineruva shobō. 